\title{
Producciones de sentido en torno a la exposición a agroquímicos. El caso de la ciudad de Young
}

\section{Productions of sense on agrochemicals exposure. The case of Young city}

\author{
Rodríguez González, Nicolás (1); Heinzen Cesio, Jimena (2). \\ (1) Instituto de Psicología Social, Polo de Salud Comunitaria, Centro Universitario Regional Litoral Norte, \\ Universidad de la República (UdelaR), Uruguay. \\ (2)Red de Atención Primaria, Administración de los Servicios de Salud del Estado (RAP-ASSE), Uruguay. \\ Contacto: nicolasr@psico.edu.uy
}

RECIBIDO: 23/3/2017 - APROBADO: 17/6/2017

\begin{abstract}
Resumen
En los últimos años Uruguay ha presentado un aumento sostenido de la producción agrícola y el uso intensivo de agroquímicos. Este nuevo escenario ha generado incertidumbres y preocupación en las comunidades expuestas a estas sustancias químicas. En este trabajo se presentan parte de los resultados de una investigación realizada en la ciudad de Young, Río Negro, Uruguay. Específicamente, se describe y analiza el modo en que la comunidad delimitó la problemática ambiental, sus sentidos sobre el territorio y los conflictos derivados de la producción agrícola extensiva. Desde un enfoque cualitativo de investigación se instrumentaron cuatro mapeos de actores sociales y cinco cartografías sociales. En ellos participaron integrantes de la comunidad, autoridades gubernamentales y actores productivos de la ciudad. Entre los resultados se destaca que la ubicación del actor en el proceso productivo condiciona las producciones de sentido en torno a los agroquímicos. Entre esos sentidos se privilegia la exposición a estas sustancias y otra serie de aspectos que son parte de la agricultura extensiva. La reproducción cotidiana de la vida aparece como uno de los condicionantes tanto de la producción subjetiva como de las posibilidades para transformar las situaciones de vulnerabilidad identificadas.

Palabras clave: Plaguicidas, percepción social, comunidad.
\end{abstract}

\begin{abstract}
Uruguay has shown an increase in agricultural production and in agrochemical use over the last few years. Communities exposed to these substances have expressed concerns and doubts about these transformations. In this article part of the results of an investigation developed in Young city, Río Negro, Uruguay, are introduced. The main purpose of this paper is to describe and analyze how ambiental problems were delimited by community members, senses about territory, and conflicts over extensive agricultural production. From a cualitative approach, four social actor maps and five social cartografies were conducted. Members of community, statal authorities and actors related to agricultural production participated in these activities. The position of actors in the productive process influences productions of sense about agrochemicals. Those senses higlight exposition to this substances and other elements that take part on extensive agriculture. Subjective production and the possibilities of transforming vulnerabilities identified appear to be conditioned by reproduction of daily life.

Keywords: Pesticides, social perceptions, community.
\end{abstract}

\section{Introducción}

Desde hace más de una década los países del cono sur tuvieron una serie de transformaciones económicas que modificaron ciertos aspectos de su matriz productiva. La producción agrícola fue una de ellas, pues se orientó fuertemente a la generación de materias primas para su exportación (Oyhancabal y Narbondo, 2009; Almeyra, et al., 2014). Estos procesos hicieron que ciertos espacios geográficos se conformaran como agrociudades, es decir, como entornos urbanos que ofician de soporte logístico, administrativo y financiero de la actividad agrícola (Hernández, et al., 2012). El avance del agrocapitalismo o agronegocio y sus cambios en los modos de producir (Morina y Cacace, 2013) motivaron la preocupación y movilización de distintos colectivos de la región -Brasil, Paraguay, Argentina y Uruguay-, en particular por sus posibles repercusiones en la salud de las poblaciones (Martínez, et al., 2006; Rulli, 2009; Palau, 2015; Abbate, et al., 2015; ABRASCO, 2015). Uno de los temas centrales es el uso intensivo de plaguicidas y agroquímicos. Estos productos adquieren distintas denominaciones de acuerdo al enfoque técnico-político del tema: agroquímicos, agrotóxicos, plaguicidas, pesticidas, etcétera (Peres y Moreira, 2003). Las comunidades y colectivos sociales también los denominan de distinto modo de acuerdo a sus 
experiencias específicas: remedio, veneno, químicos, etcétera (Castro y Confalonieri, 2005; Gutiérrez-Strauss, et al., 2013; Vargas Trejos, 2015). A los efectos de este trabajo se utilizará el término agroquímicos, porque aunque no se desconoce su toxicidad humana y ambiental, se entiende que esta depende de sus condiciones de uso, lo cual es un elemento que está estrechamente ligado al modelo productivo dominante (Rodríguez, 2017). La producción de mercancías ante cualquier otro objetivo es una de las transformaciones que el agronegocio introdujo en el espacio rural latinoamericano (Mançano, 2013; Almeyra, et al., 2014). En este contexto, el uso intensivo de agroquímicos es una de las estrategias utilizadas para maximizar los resultados productivos y económicos, más allá de los riesgos y vulnerabilidades para la salud humana que han sido identificados (Ritter, et al., 2006; Alavanja y Bonner, 2012; Ye, et al., 2013; Blair, et al., 2014; ABRASCO, 2015).

En Uruguay, a pesar de la existencia de marcos normativos que regulan la utilización de estas tecnologías (Neme, et al., 2010), la expansión de la frontera agrícola a zonas eminentemente urbanas movilizó acciones de distintas comunidades, principalmente por sus posibles impactos sanitarios y ambientales (Martínez, et al., 2006; Ríos, et al., 2010; Abbate, et al., 2015). Este fue el caso de un colectivo de la ciudad de Young, Río Negro, Uruguay, que en 2012 transmitió a un equipo de investigadores de la Universidad de la República (UdelaR) ciertas inquietudes vinculadas al tema. Gracias a una primera aproximación a la problemática, se acordó con integrantes de la comunidad la necesidad de avanzar en el diseño de un monitoreo ambiental y laboral en el territorio. Para tal fin se diseñó la investigación «Salud laboral y ambiental en torno a la utilización de agroquímicos en la ciudad de Young: estrategias de monitoreo participativo", que tuvo el cometido de conocer el estado de situación en cuanto a la utilización de agroquímicos, los procesos peligrosos para la salud y la generación de instrumentos que permitieran registrar, controlar y eventualmente eliminar o paliar sus efectos. Desde una perspectiva de salud colectiva, la investigación se orientó a democratizar el saber científico con respecto al tema agroquímicos, así como a socializar los resultados de la situación de la ciudad de Young, la producción dominante y los procesos peligrosos para la salud (Abbate, et al., 2015). El análisis de las vulnerabilidades para la salud fueron considerados en sus dimensiones individuales (los tipos de exposición y las sustancias predominantes), colectivas (las formas de producción, el hábitat y el uso del espacio público, la producción social de sentido), y contextuales o programáticas (sistema de salud, campañas educativas, normativas) (Ayres, et al., 2009). En este trabajo se presenta lo referido a la primera parte de la investigación, que estuvo dirigida a conocer a los actores de la ciudad y los sentidos que producen sobre los agroquímicos y sus vulnerabilidades ambientales y sanitarias. Específicamente, se describe y analiza el modo en que la comunidad delimitó la problemática, sus sentidos y significaciones sobre el territorio, y los conflictos derivados de la producción agrícola extensiva.

La consideración del territorio como construcción social del espacio geográfico (Mançano, 2013) implicó reconocer y estudiar las producciones de sentido que los sujetos crean en torno a las relaciones materiales que en ese espacio se establecen. Estas no necesariamente se rigen por criterios de verdad ni de cientificidad. Las producciones de sentido son el resultado de la capacidad creativa de los sujetos, que en el campo de lo histórico social se denomina como imaginario social (Castoriadis, 2013). Esas producciones emergen de las relaciones de poder que acontecen en cierto contexto histórico y geográfico. Cuando esas producciones de sentido tienden a lo establecido y lo conservador son el resultado del imaginario social instituido; mientras que cuando lo hacen en un sentido crítico y transformador son el producto del imaginario social instituyente (Reygadas, 1998; Fernández, 2007). Su diferencia radica no en un criterio axiológico, sino en los modos en que los sujetos significan la realidad en un contexto social específico. En la investigación que se desarrolla en este trabajo fueron consideradas las significaciones sociales producidas sobre la exposición colectiva y comunitaria a los agroquímicos. Se partió de entender al territorio como habitáculo de significaciones diversas, donde sus múltiples actores producen relatos, vivencias y opiniones de distinto tipo. Concebir a las producciones de sentido desde lo múltiple y no desde lo uno supuso resignarse a la generación de explicaciones totalizadoras y a concebir las creaciones colectivas de significados como partes entre las partes (Guattari, 1996; Álvarez, 2014). Por ende, cuando se refiere a la comunidad de Young, no se la concibe como algo homogéneo, único y exento de conflictos, sino como algo diverso y que produce distintas construcciones sobre el mismo espacio geográfico.

Los estudios sobre las relaciones entre producción de subjetividad y exposición laboral y comunitaria a los agroquímicos se han multiplicado desde 1990 a nuestros días. Utilizando distintas categorías teóricas para referirse a lo subjetivo -percepción social, creencias, representaciones sociales-, durante 30 años y con distintas intensidades se han investigado las creaciones sociales de productores, trabajadores rurales y comunidades (Rodríguez, 2017). Estas investigaciones se dedicaron mayoritariamente a los actores directamente vinculados con lo productivo, es decir, agricultores y trabajadores, y son relativamente escasos los trabajos que abordan comunidades con múltiples actores (Ríos-González, et al., 2013). Entre estos últimos se destacan los trabajos de Arellano et al. (2009) en dos territorios del Bajo California, México, donde las diferencias en la percepción de riesgos para la salud estuvieron condicionadas por el lugar geográfico y sus trayectorias productivas, y por el nivel de escolaridad de los participantes del estudio. La investigación de Barraza et al. (2011) en plantaciones de banana en Costa Rica es otro antecedente en este sentido; los autores señalan que las diferencias en la percepción de riesgo están condicionadas por el modelo productivo implementado en la zona (producción familiar o cultivos extensivos), la posición del sujeto en el proceso agrícola y su condición de género. Un estudio posterior de Ríos-González et al., (2013) en el sur de México reafirma la relación entre percepción del riesgo en torno a los agroquímicos y la ubicación del actor en el proceso productivo. Estos antecedentes permiten observar las complejidades involucradas en el estudio de las producciones colectivas de sentido y los múltiples elementos que las condicionan. Elucidar estos elementos en la agrociudad de Young fue parte de los propósitos de esta investigación.

\section{Materiales y Métodos}

Para poder realizar una primera caracterización del estado de situación en torno a la utilización de agroquímicos fue necesario delimitar el área y la población que formaría parte del estudio. El área se definió con un criterio de densidad poblacional y quedó limitada a la ciudad de Young y su en- 
torno geográfico $(15 \mathrm{~km})$. Según datos del Censo Nacional (INE, 2011), allí residen 16.756 personas. El trabajo que se presenta puede caracterizarse como un estudio de caso, dado que se tomó un caso particular, la comunidad de la ciudad de Young y su entorno geográfico, con el objetivo de alcanzar una comprensión integral del problema de investigación. Los estudios de casos permiten producir conocimiento novedoso a partir de una delimitación teórica de la situación y sus posibilidades de generalización analítica a otros contextos. En cuanto al diseño, los estudios de casos han sido caracterizados con cierta independencia de las clasificaciones tradicionales de diseños experimentales/ no experimentales o diseños cuantitativos/ cualitativos/mixtos. Este tipo de investigación puede utilizar herramientas cuantitativas, cualitativas o mixtas, así como incluir experimentos o bien ser observacionales, dado que lo que los define es la selección de una o varias unidades que serán analizadas en profundidad para lograr responder al problema propuesto (Stake, 1999; Vasilachis, 2007).

En esta investigación se realizó un muestreo intencional teórico (Vasilachis, 2007) o de casos-tipo (Hernández, et al., 2006), donde el criterio de selección de las personas estuvo dado por su condición de informantes clave o conocedores de los temas de interés (Montero, 2006). En tanto se pretendió comprender las producciones de sentido sobre el territorio y las problemáticas derivadas de la utilización de agroquímicos, la muestra integró a jóvenes y adultos de la ciudad de Young que tuvieran algún tipo de interés en conocer el estado de situación en relación a estas sustancias químicas, y que procedieran de distintos estratos y ámbitos sociales: trabajadores rurales, autoridades nacionales y municipales, empresarios/as, técnicos/as e integrantes de organizaciones sociales. Así, la muestra quedó conformada por aquellas personas que participaron de las distintas instancias de investigación. Este muestreo de tipo cualitativo estuvo dirigido a conocer en profundidad el modo en que esta diversidad de actores concebían la situación de los agroquímicos y los procesos de salud-enfermedad en el territorio. Como se desarrolló previamente, los estudios de casos buscan comprender un fenómeno en profundidad, lo que implica conocer el problema en sus particularidades y singularidades. Las generalizaciones a otros escenarios se pueden realizar en términos analíticos, pero no es un objetivo de este tipo de diseños (Vasilachis, 2007).

Para la aplicación de técnicas cualitativas se definieron categorías operacionales a partir de las cuales se realizó la recolección de datos. Estas categorías son elaboradas a priori con el objetivo de aproximarse al objeto de la investigación y son desarrolladas específicamente para la observación de un determinado objeto de estudio (Minayo, 2009). A partir de ellas se realiza un primer nivel de análisis descriptivo; en este estudio se definieron las siguientes: a) actores presentes en la ciudad y afinidad con la temática; b) conflictos ambientales en el territorio; c) exposición a agroquímicos y poblaciones vulnerables, y d) problemas derivados de la aplicación de agroquímicos. A partir de estas categorías operacionales (Minayo, 2009) se relevaron las producciones de sentido colectivas en torno a las vulnerabilidades derivadas de la utilización intensiva de agroquímicos.

En cuanto al diseño, se realizó un estudio transversal de características exploratorias, dado que no se identificaron antecedentes nacionales de investigaciones participativas que abordaran esta temática. Para cumplir los objetivos propuestos en esta etapa de la investigación se optó por técnicas provenientes de los enfoques cualitativos de investigación (Hernández, et al., 2006; Vasilachis, 2007; Minayo, 2009). En particular se privilegiaron dos técnicas grupales de investigación: los mapeos de actores sociales (Blanco, 2010) y las cartografías sociales (De Castro, 1997).

La técnica de mapeo de actores sociales permite identificar, caracterizar y categorizar a las instituciones, organizaciones o personas particulares que están presentes o que inciden en una zona, en forma directa o indirecta. Los actores sociales pueden definirse como todos aquellos colectivos con capacidad de construcción de la realidad social y del territorio (Blanco, 2010). Esta técnica se aplicó en la primera etapa de la investigación y buscó cumplir con dos objetivos: por un lado, dar difusión al proyecto de investigación y sus objetivos principales, y por otro, identificar actores afines al desarrollo del proyecto, así como posibles amenazas u obstáculos. Se trata de una técnica participativa en la que se parte de un diagrama en blanco en el que se ubica a nivel central el objetivo de la investigación, posteriormente se dibujan tres círculos concéntricos a partir del objetivo. Desde el equipo de investigación se explicó la consigna a los participantes, que consistió en identificar actores sociales de la ciudad y situarlos en función de su cercanía o lejanía con el tema en alguno de los tres círculos. Los actores institucionales fueron representados con un cuadrado, las organizaciones sociales con círculos y los sectores empresariales con triángulos. Posteriormente, se identificó la afinidad con el tema según una escala: muy afín ++, afín +, regular +/-, distante -, muy distante - También se solicitó definir el nivel de «poder» en relación a la temática de cada actor, en este caso se resaltaba el actor en función del poder: poco poder un solo margen, moderado poder dos márgenes y mucho poder tres márgenes (Villasante y Gutiérrez, 2007; Rance y Tellería, 2003). Esto permitió reconocer a personas, grupos e instituciones, y establecer su afinidad con la temática de estudio, así como su nivel de injerencia o poder de intervención, según los participantes de los talleres de investigación (Blanco, 2010). Se realizaron un total de cuatro mapeos de actores sociales, tres de ellos en ámbitos que ya existían en la comunidad de Young y otro convocado por el equipo de investigación: Comisión Pro Universidad de Young, Mesa de Desarrollo Rural, Mesa de Articulación de Políticas Sociales y actores vinculados al sistema local de salud. Simultáneamente a la discusión y representación gráfica del mapa se completó una planilla en la que se registró: actor social, área de influencia, participantes, afinidad con el tema, conflictos y observaciones que surgían de la discusión entre los participantes en torno al actor social. A partir de un primer nivel de análisis de los resultados obtenidos se definieron grupos con los cuales instrumentar las cartografías sociales.

El objetivo de las cartografías fue comprender las producciones de sentido de la comunidad en torno al territorio, los conflictos ambientales y las poblaciones vulnerables. La cartografía es una técnica participativa en la que, a través de mapas y preguntas orientadoras, los participantes identifican distintos espacios de relevancia según la temática en cuestión (De Castro, 1997). En el caso de la investigación que se presenta las cartografías sociales buscaron cumplir con el objetivo de reconocer espacialmente la distribución y desarrollo de la producción local y su relación con el hábitat, así como guiar la toma de muestras para el «monitoreo químico» de agroquímicos en aguas y colmenas. Para la ins- 
trumentación de esta técnica se partió de un mapa en blanco de la zona delimitada con escasas referencias que permiten ubicar a los participantes en el espacio geográfico. A través de íconos que representaban distintos procesos productivos (por ejemplo, ganadería, forestación, agricultura), elementos vinculados a la producción (por ejemplo, silos, camiones, envases de agroquímicos) y marcadores de colores, se invitó a los participantes a colocarlos en el mapa o bien a dibujar elementos que no habían sido considerados. Asimismo, para favorecer el intercambio en torno al espacio geográfico se propusieron tres preguntas disparadoras: 1) ¿dónde se encuentran los conflictos o problemas vinculados a las formas del sistema productivo?, ¿de qué tipo son?; 2) ¿existen poblaciones vulnerables en torno a las áreas de conflicto?, por ejemplo, referidas a fumigaciones con agroquímicos: escuelas, poblaciones rurales, etcétera; 3 ) ¿existen zonas problemáticas en cuanto al desecho de agroquímicos?, por ejemplo, por lavado de maquinaria agrícola o descarte de envases en zonas no adecuadas.

Se realizaron cinco talleres utilizando la cartografía social como técnica: colectivo de profesionales y docentes nucleados en la Comisión Pro Universidad de Young, apicultores que integraban una cooperativa de producción, estudiantes de secundaria y de enseñanza técnica, y población en general. En ambas técnicas de investigación colectiva el equipo interdisciplinario moderó los intercambios e interrogó sobre los elementos que no quedaban expresados de forma clara o que era necesario profundizar. Todas las instancias se registraron con audio digital y su contenido fue transcripto.

Entre 100 y 150 personas formaron parte del proceso de investigación y colaboraron alrededor de 35 organizaciones de distinto tipo: empresariales, sociales, educativas, etcétera. El procesamiento del material emergente se realizó in situ durante el proceso de investigación, para poder identificar la saturación de información o de categorías (Hernández, et al., 2006; Vasilachis, 2007), es decir, se observó cuándo la técnica no aportaba nuevos elementos o información adicional a la ya recabada. Para ese procesamiento se realizó un análisis de contenido donde se identificaron los núcleos de sentido del material cualitativo y se construyeron categorías analíticas para comprender el fenómeno de estudio (Minayo, 2009). Las categorías analíticas o empíricas permiten profundizar en la comprensión del objeto de estudio, surgen de la realidad empírica observada, como su nombre lo indica, y son, por tanto, elaboraciones de los investigadores en la búsqueda de aprehender y comprender el problema de investigación (Minayo, 2009). Estas categorías permitieron construir una tipología de los actores de la ciudad, identificar el vínculo con la temática de los agroquímicos, observar las producciones de sentido ambientales y sanitarias, y delimitar los elementos relacionales para el diseño de un monitoreo participativo. Durante todo el proceso de investigación se respetó la normativa regulada en el Decreto 379/008 (Uruguay, 2008) sobre investigación con seres humanos. A tales efectos los participantes firmaron un consentimiento informado que detallaba los objetivos del estudio, la garantía de resguardo de su confidencialidad, la posibilidad de participar voluntariamente así como de retirarse si lo consideraban oportuno, y los contactos de los responsables de la investigación. El proyecto en su totalidad fue evaluado y aprobado para su implementación por el Comité de Ética de la Investigación de la Facultad de Psicología de la Universidad de la República (Exp. No 191130-000695-12).

\section{Resultados}

Por un lado, para la presentación de los resultados obtenidos se desarrolla en esta sección lo que surgió de los mapeos de actores sociales, ya que este nivel de resultados hacen a la aproximación inicial a la problemática. En particular, se describen cuáles son los actores y sus posibilidades de injerencia en la temática propuesta por este proyecto. Por otro lado, se detalla lo emergente de las cartografías sociales en tanto esto constituye otro nivel de resultados, más específicos, sobre las situaciones de vulnerabilidad y los conflictos vinculados con la utilización de agroquímicos. De este modo, primero se exponen los actores, su nivel de poder en la comunidad y la afinidad o no con la temática de estudio, y posteriormente se presentan las producciones de sentido vinculadas con las dimensiones urbanas de la producción agrícola, las significaciones sobre la producción rural, las poblaciones vulnerables identificadas por la comunidad de Young, las implicaciones de los vínculos interpersonales en el territorio, y las distintas interrogantes que se presentaron durante la ejecución de esta investigación. Se enfatizan los resultados en sus aspectos descriptivos, pues más adelante se discuten y analizan críticamente a la luz del conocimiento social disponible.

\section{Agroquímicos, actores y poder}

En cuanto a los actores colectivos que tenían algún nivel de vinculación con el tema agroquímicos, se logró diferenciar instituciones gubernamentales de organizaciones sociales y organizaciones empresariales. Los trabajadores rurales y el personal de salud aparecieron como actores específicos, independientes de las organizaciones o instituciones que los nuclean (Mapeo No 4; los mapeos de actores y las cartografías sociales fueron numeradas en el orden cronológico en que fueron realizados para resguardar la confidencialidad de los informantes). Sobre la afinidad de los actores identificados con el análisis de la situación de los agroquímicos, son diversas las asignaciones o adjudicaciones de responsabilidad o sensibilidad en cuanto al tema. En las instituciones gubernamentales se observan afinidades, pero con expresión a nivel territorial-local, lo cual no siempre se corresponde con las directrices nacionales. Entre los actores locales afines se identifica al Ministerio de Ganadería, Agricultura y Pesca, la Administración de Servicios de Salud del Estado y la Administración Nacional de Educación Pública. El municipio de Young es identificado como un actor afín a la investigación pero con limitaciones en relación con la Intendencia de Río Negro, de la cual depende. Al conjunto de actores gubernamentales se lo señala con injerencia en el tema por su comunicación y legitimación a nivel de la población (Mapeo $\mathrm{N}^{\circ} 1$ ).

Con respecto a las organizaciones empresariales, se menciona a una diversidad de empresas con distintos cometidos en lo que refiere a la producción agrícola. En los mapeos se las reconoce con poder económico y político, en particular porque algunas de ellas son multinacionales del agronegocio (Mapeo $\mathrm{N}^{\circ} 1$ ). En relación a la afinidad con el tema, los actores extra-productivos manifiestan que las empresas no serían afines a investigar la situación de los agroquímicos, e inclusive se expresaron reparos al momento de nombrarlas, por el poder y control que tienen sobre el territorio (Mapeo No 4). Los actores productivos sí se autoidentifican como afines a investigar el tema, pero la afinidad se concentra principalmente en los productos que se utilizan y en sus posibles impactos 


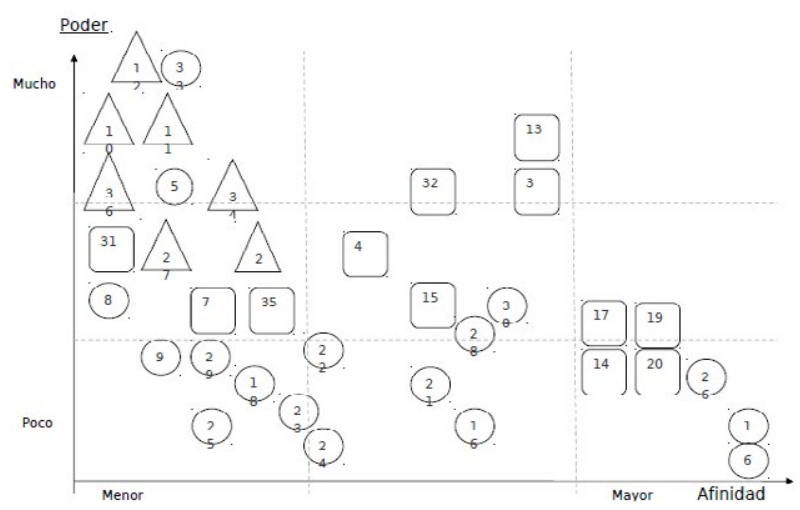

Figura 1. Esquema gráfico de mapeo de actores sociales No 1. Elaborado por Andrés Fonsalía.

en la producción (Mapeo No 2). Este elemento aparece como transversal a los pequeños y grandes productores.

Finalmente, las organizaciones sociales de corte popular fueron identificadas como afines a la temática pero con escasa injerencia en su transformación concreta. Entre ellas, se menciona a las organizaciones de usuarios de la salud, los grupos ecologistas y a los trabajadores organizados. Se señala que el tema tiene un tiempo de discusión en la ciudad pero que el debate se ha dado de forma fragmentaria (Mapeo $\mathrm{N}^{\circ} 3$ ). A los trabajadores rurales se los identifica como afines con la temática pero con escasa capacidad de colaboración, en particular por el riesgo de perder su empleo (Mapeo № 4). Por su parte, el personal de salud se manifestó con interés en la temática pero hasta el momento de la investigación nunca había desarrollado actividades o algún programa relacionado con la exposición a agroquímicos.

En la Figura 1 se representa lo surgido en uno de los mapeos de actores sociales, que sintetiza a nivel general lo expresado anteriormente en cuanto a tipo de actores, nivel de poder y afinidad con la temática de investigación. Con un círculo están representadas las organizaciones sociales, con un cuadrado los organismos y dependencias públicas, y con un triángulo las empresas.

\section{Aspectos urbanos de la producción agrícola}

Los aspectos urbanos de la producción agrícola hacen a la convivencia de la población de Young con los diferentes cultivos de la zona. Se destacaron distintos elementos relativos a la producción agrícola, tanto a lo estrictamente productivo como a toda la logística necesaria para que ésta se desarrolle. Un primer elemento común de preocupación fue el tema del tránsito en algunos ejes viales del centro urbano. Se manifestó que el tránsito de camiones que transportan granos e insumos es significativo sobre todo en época de zafra. Al respecto se expresó que se da por dentro de la ciudad, sin zonas ni horarios de exclusión: «Camiones ponemos por todos lados» (Cartografía No 3). En cuanto a este tema no se registró un consenso en las diferentes cartografías. Algunos participantes plantearon que la circulación de camiones se encuentra asociada a los silos, y que estos transitan fundamentalmente por las vías perimetrales. Sin embargo, otros expresaron que los camiones suelen transitar por la ciudad, desde las rutas y avenidas principales hasta las calles y caminos vecinales. Un segundo elemento vinculado a la producción estuvo dado por la maquinaria de aplicación terrestre de plaguicidas o «mosquitos». Se manifestó que transitan por la ciudad, y se mencionaron casos que son estacionados y lavados en barrios densamente poblados. Las menciones a espacios de la ciudad donde se convive con maquinaria utilizada para fumigación fueron numerosas y diversas: «Está todo lleno de mosquitos, desparramá por todos lados mosquitos, está lleno» (Cartografía № 2). Las referencias identifican la circulación, estacionamiento, mantenimiento y lavado de «mosquitos» en las inmediaciones de viviendas, escuelas y comisarias, entre otros espacios. También fueron mencionadas situaciones donde los «mosquitos» se lavan en los cauces de agua y en establecimientos donde la eliminación de los residuos es hacia la calle. Ante esto se destaca la presencia de olores desagradables en los lugares que se estacionan y/o lava esta maquinaria de aplicación. Por último, otro aspecto relacionado a la convivencia urbana con la producción agrícola está dado por la cercanía de los cultivos, que fueron identificados rodeando la ciudad. A pesar de los distintos cultivos agrícolas presentes, cuando se hacía alusión a ellos solo se identificaba la soja. Más adelante se mencionan los otros rubros productivos que fueron reconocidos, pero, en lo que a producción agrícola refiere, el cultivo de soja se observa como dominante. Además de la proximidad efectiva de las plantaciones, los medios que hacen posible la producción están plenamente integrados a la ciudad. Además de los camiones y maquinaria agrícola también se señaló la presencia de silos y centros de acopio, así como los insumos agropecuarios y el desecho de envases de agroquímicos. Las empresas que venden insumos, maquinaria y agroquímicos son diversas y están presentes en toda la ciudad. Los silos y centros de acopio fueron descriptos como espacios problemáticos por el polvillo que generan, la circulación de camiones y maquinaria y el descarte de envases. Son diversas las menciones a la ubicación de los silos y se destaca la alusión a que su ubicación espacial no se dio de forma planificada, o que el desarrollo de la ciudad fue avanzando hasta su entorno.

\section{Sentidos sobre la producción rural}

Al momento de identificar las distintas producciones presentes en Young y su entorno surgen numerosos emprendimientos productivos, en su mayoría vinculados a lo agroindustrial. Se enumeran las distintas producciones identificadas y luego se analiza el sentido que se le asignó a cada una de ellas en las distintas cartografías: a) cereales, b) forestación, c) ganadería, d) apicultura y e) lechería. Como ya fue mencionado, quienes participaron de las cartografías identificaron la soja como el cultivo con mayor presencia en la zona; son mínimas las menciones a otros tipos de cultivos, ya sea de invierno, como el trigo, o que conviven con la soja, como el sorgo. Los diferentes grupos señalaron extensas zonas de los campos adyacentes a la ciudad como zonas de cultivo de soja: «soja pone por todos lados, estamos rodeados» (Cartografía № 2); «ila soja está en todos lados!» (Cartografía No 3). Quienes traen a colación la presencia de otros cultivos son personas vinculadas al sector a través de su trabajo, mientras que los vecinos más alejados al tema tienden a mencionar a la soja como el único cultivo. Además de los cultivos extensivos, se señalaron los elementos que se relacionan con ella en la ciudad, tal como ya fue descrito anteriormente: silos, galpones de acopio de granos y de envases de agroquímicos. En relación a estos últimos, se señaló que su limpieza y eliminación no ha sido completamente planificada. Si bien existen distintos proyectos paliativos del problema de 
los envases vacíos y reglamentación respecto a su descarte, los participantes de los distintos talleres manifiestan que los envases de agroquímicos pueden encontrarse en distintos espacios de la ciudad. En el momento en que se estaba ejecutando esta etapa del estudio se relató que era frecuente encontrar envases en predios de chatarrerías, baldíos, cauces de arroyos, galpones, etcétera. La forestación es otro de los sectores productivos que se identificó, si bien las zonas de mayor concentración no se encuentran dentro del área delimitada para el estudio $(15 \mathrm{~km}$ de la ciudad). Durante el espacio de taller se plantearon dudas en torno a la ubicación exacta de los predios forestados, presentes por su incidencia en la dinámica urbana, aunque no se convive con ellos directamente. La ganadería fue menos mencionada como rama de actividad; uno de los participantes justificó que «quedan pocas vacas» (Cartografía No 3). Otro participante explicó que «la ganadería coexiste con la agricultura pero hay mucho más agricultura» (Cartografía № 2). En cuanto a la apicultura, se reconoció como proceso productivo presente en la ciudad. Se mencionó que por la nueva reglamentación de ordenamiento territorial las colmenas ya no se pueden ubicar en la ciudad. En la ciudad de Young hay aproximadamente 200 apicultores, de los cuales 90 se encuentran nucleados en la Cooperativa Agraria Limitada Apicultores de Young (CALAY). La lechería está presente en la zona rural a través de pequeños establecimientos tipo tambos, mientras que en la ciudad una empresa tiene una presencia significativa por ser una industria con capacidad de producción para la distribución a nivel nacional e internacional.

\section{Manejo de agroquímicos y poblaciones vulnerables}

Un primer elemento expresado en cuanto al manejo de agroquímicos fue lo que refiere a su venta. Se mencionaron distintas situaciones en las que han habido derrames y los comercios linderos a los establecimientos han hecho denuncias. A su vez, se señaló que la venta de estos productos no está organizada en el espacio, ya que se comercializan en veterinarias, al lado de escuelas, etcétera.

En segundo lugar, otra problemática identificada refiere a lo que sigue a la aplicación de los productos y al desecho de los envases vacíos, como ya fue señalado anteriormente. La periferia de la ciudad aparece como espacio vulnerable con respecto a este tema, pues se identificaron diferentes lugares donde se pueden encontrar desechos de envases de agroquímicos. Se mencionó el proyecto Campo Limpio como lugar de acopio de estos envases, pero también que se encontraba desbordado y que no estaba pudiendo cubrir toda la demanda. Un participante agregó que otro gran problema son los productos vencidos, ya que ni la Dirección Nacional de Medio Ambiente ni el Ministerio de Ganadería, Agricultura y Pesca tienen previsto el desecho de estos productos.

En tercer lugar, se visualizó que ciertas áreas de esparcimiento público que poseen cursos de agua pueden estar afectadas por el manejo de agroquímicos para la producción: Don Esteban, Las Piedras, Cañada de los Chanchos y Arroyo Santa María. Se identificó a esos lugares como zonas de riesgo porque son espacios de baño y pesca (Cartografías No 1,2 y 5). En cuanto a las poblaciones vulnerables fue dominante la alusión a las escuelas rurales. También se las reconoció como zonas de conflicto ambiental porque han habido denuncias judiciales por fumigaciones. Además de la fumigación directa, se identificó como posible problema la contaminación de los lugares donde se extrae agua para el consumo de los niños y niñas. En lo que refiere a escuelas del área urbana se mencionó la cercanía con locales de venta de productos químicos y con los silos de acopio de granos. Asimismo, fueron identificadas áreas de contaminación ambiental que exceden a los agroquímicos. Entre ellas se destacaron las piletas de decantación del saneamiento, aunque se mencionaron las mejoras que esto supone para las condiciones urbanas. Algunas de ellas están en construcción y se visualizan como potencialmente conflictivas. Otro elemento señalado fue la utilización de agua de pozo para el abastecimiento urbano de agua corriente. Un arroyo en particular generaba preocupación y se lo identificó como un lugar que podría estar contaminado. Allí, además de decantar el saneamiento, llega el desagüe de la industrial láctea y es una zona que está rodeada de cultivos agrícolas.

\section{Obstáculos de la proximidad interpersonal}

En distintos actores se pudo constatar la preocupación por el impacto del uso de los agroquímicos en la población y ambiente. Sin embargo, las tensiones y contradicciones que estas interrogantes generan no se explicitan en la esfera de lo público. Quienes manifestaron dudas o reparos sobre el modelo productivo suelen hacerlo en confianza, en ámbitos familiares o reducidos. Se identificó la cercanía y la proximidad de la población de Young como uno de los obstáculos para explicitar de forma manifiesta los malestares e interrogantes. Es decir, el hecho de que Young cuenta con una población relativamente pequeña, en la que la mayor parte de las personas se conocen o tienen cierto relacionamiento, debilita la expresión del conflicto territorial en la esfera de lo público. Entre las referencias que los participantes de la cartografías hicieron al tema se destacan: «acá es muy chiquito y nos conocemos todos» (Cartografía No 3 ), y «las mismas personas estamos en tantas cosas a la vez que nos genera conflicto ético» (Cartografía No 2). A esto se añaden los señalamientos en torno a la "falta de conciencia» de la gente (Cartografía No 3). La proximidad de los vínculos fue uno de los elementos señalados que se complementa con la dimensión económica del proceso: el crecimiento de Young de los últimos años está fuertemente asociado al agronegocio, a través del cual, directa o indirectamente, las familias han mejorado sus ingresos. Esto fue claramente graficado en uno de los talleres: «la gente no va a criticar lo que le da de comer» (Cartografía № 2).

\section{Interrogantes sobre la producción}

En las distintas instancias grupales instrumentadas a lo largo de la investigación se manifestaron distintas interrogantes que fueron contestadas entre los participantes o que quedaban planteadas como dudas. Algunas de ellas estaban vinculadas al ordenamiento territorial y a la normativa existente en relación a la aplicación de agroquímicos:

- ¿El área de exclusión para aplicaciones abarca a toda la ciudad o solo a la zona urbana?

- ¿La disposición reglamentaria de 300 metros para aplicaciones terrestres y 500 metros para las áreas también aplica para las escuelas rurales?

Otras interrogantes estuvieron vinculadas con el tipo de semillas que se utilizaban y los posibles riesgos para los 


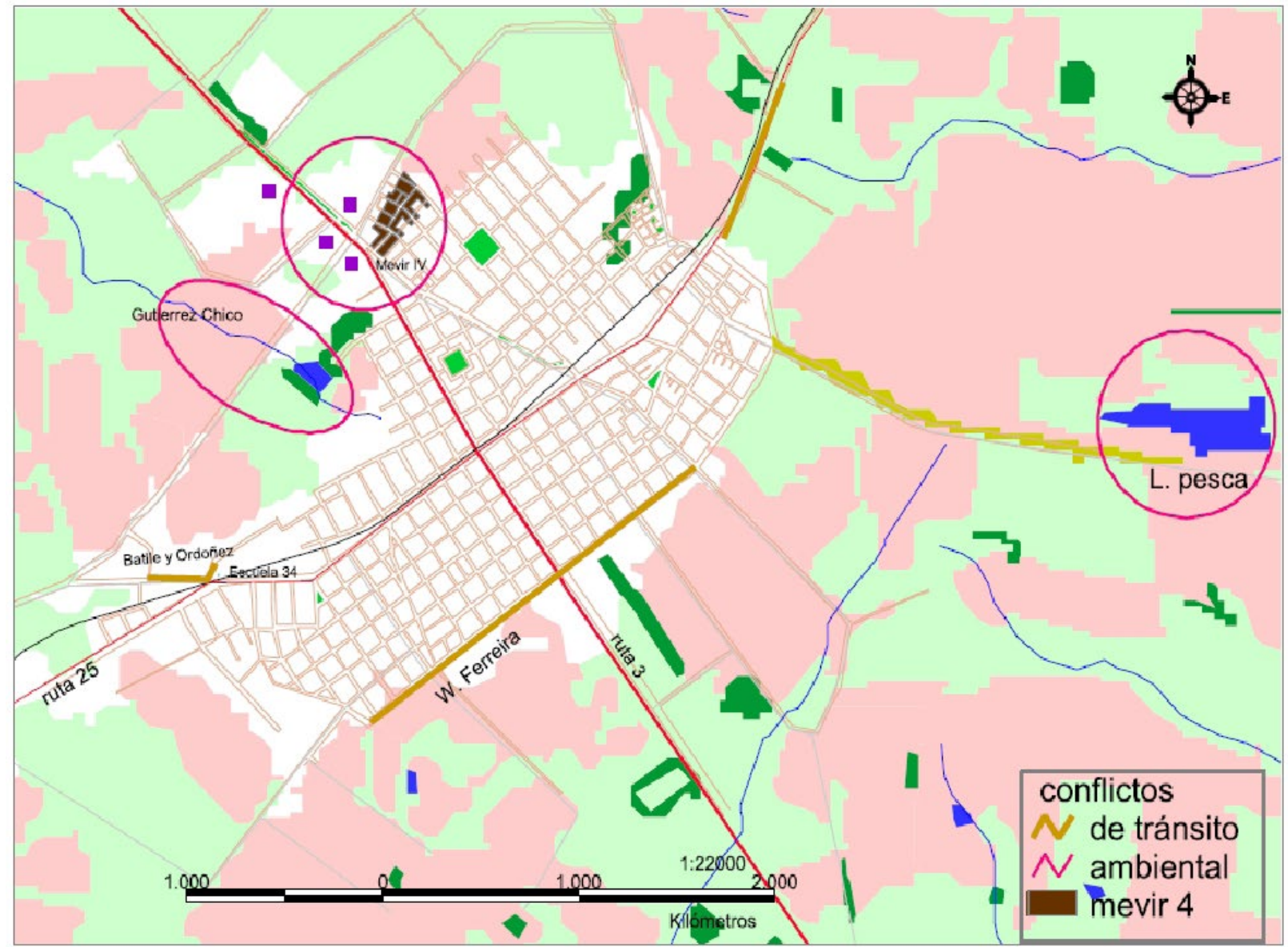

Figura 2. Mapa síntesis con datos surgidos en las cartografías sociales. Fuente: Abbate, et al., 2015.

otros tipos de producción, en particular para la apicultura. Se mencionó que se estaba cultivando un nuevo tipo de soja que no requiere la aplicación de agroquímicos, pero se desconocía su uso específico y si afectaba la producción de miel (Cartografía No 2). Por último, se expresaron preocupaciones por el consumo humano de leche vacuna, en tanto los animales conviven con los campos agrícolas y beben agua de las cañadas que los rodean.

En la Figura 2 se presenta un mapa de la ciudad de Young que contiene una síntesis de los resultados de las cinco cartografías sociales que se realizaron. En él se encuentran señaladas las zonas de conflicto ambiental con un círculo rosado. Entre esas zonas se identificó al barrio Mevir 4 como una población particularmente vulnerable. En la figura están referenciadas en amarillo las vías de tránsito pesado y de maquinaria agrícola.

\section{Discusión y Conclusiones}

El proceso de investigación permitió, por un lado, conocer los actores presentes en la agrociudad de Young, su afinidad e injerencia en cuanto al uso de agroquímicos. Por otro lado, se pudo objetivar con mayor precisión la relación entre el territorio y las producciones de sentido que sobre él se producen, con especial atención en lo referido a los agroquímicos y su vínculo con los procesos productivos. En la ciudad se pudieron identificar una diversidad de actores e intereses en cuanto al estudio de la temática. Las fundamentaciones del interés eran distintas, así como las posibilidades de incidir en los procesos peligrosos visualizados. Los actores estrictamente sociales (organizaciones gremiales, grupo ecologista, población en general) eran los que manifestaban mayor afinidad con el estudio de las situaciones derivadas del uso de agroquímicos, pero consideraban tener escaso nivel de injerencia en el tema. Por su parte, los pequeños productores y los actores gubernamentales presentaban una posición dual ante los agroquímicos, en los primeros por la dependencia-afectación de la producción agropecuaria de la zona, y en los organismos públicos por las diferencias entre las directrices locales y nacionales. Finalmente, los actores empresariales mostraron afinidad con el tema pero en sus aspectos productivos, y si bien pueden tener incidencia en las situaciones peligrosas, en el estudio no se plantearon indicios de posibles modificaciones en las prácticas agrícolas dominantes.

Además de la elucidación de estos elementos, la investigación posibilitó dimensionar la problemática ambiental y las poblaciones vulnerables a la utilización de agroquímicos y a otros componentes del modelo productivo. Un elemento que pudo ser observado fue que en este tipo de ciudades agroindustriales los agroquímicos son una de las tantas tecnologías utilizadas para la producción agrícola y su soporte logístico: maquinaria, transporte, acopio de granos y envases. Los actores sociales locales reconocieron diversas situaciones de riesgo en el manejo de los agroquímicos y la vulnerabilidad 
de ciertos grupos poblaciones, en particular los que residen en la zona periférica de la ciudad. Dicha situación estaba determinada por un mayor contacto con la aplicación de agroquímicos, por el tránsito de trasporte pesado y por la cercanía con centros de acopio de granos o silos. Más allá de la identificación de estos procesos peligrosos se señalaron dificultades para plantearlos públicamente, ya que la proximidad interpersonal y la dependencia económica con el modelo productivo aparecieron como elementos condicionantes. Por último, los cambios productivos acontecidos desde hace más de una década y la normativa generada para paliar sus efectos todavía genera interrogantes entre la comunidad, y en torno a ellas se producen significaciones sociales que vienen a cubrir esos vacíos o brechas de certezas.

Sintetizados los principales resultados, corresponde detenerse en tres niveles de análisis y problematización de lo emergente en este proceso colectivo de investigación. En primer lugar, es posible observar que las producciones de sentido sobre el uso de agroquímicos están estrechamente ligadas con la configuración del territorio y sus procesos productivos. Los cambios acontecidos en el sector agrícola uruguayo fueron particularmente paradigmáticos en la zona litoral oeste (Oyhancabal y Narbondo, 2009). En la ciudad de Young este modelo imprimió ciertas dinámicas urbanas, donde la producción parece invadirlo todo, tal como fue señalado en relación a la soja o a la maquinaria de aplicación terrestre de agroquímicos, los «mosquitos». Estas producciones subjetivas en algunos casos concuerdan con lo que efectivamente sucede en el territorio y en otros vienen a cubrir una brecha de sentido o incertidumbre comunitaria. Deslegitimarlas en función de criterios de veracidad o de cientificidad no posibilita partir del «punto de vista» de los sujetos involucrados (Menéndez, 2010) y comprender los condicionantes de esas producciones de sentido. Durante el proceso de investigación se pudo observar cierta discordancia entre el impulso estatal y empresarial del agronegocio como modelo agrícola privilegiado, y el involucramiento de la sociedad civil en el seguimiento y evaluación de los procesos peligrosos que ese modelo implica (Abbate, et al., 2015). Esto puede permitir comprender las brechas de sentido anteriormente señaladas.

En segundo lugar, es posible observar entonces una producción de subjetividad que se enlaza con los procesos de reproducción social de la vida que acontecen en el territorio. Samaja (2009) sostiene que esa subjetividad es la que mediatiza los distintos estratos o niveles de reproducción social. Para su estudio diseñó un esquema interpretativo de la interacción que acontece entre los procesos productivos y reproductivos de la vida social. Según el autor, existen cuatro niveles de la reproducción social: a) biocomunal (familia biológica), b) comuno cultural (sistema cultural), c) político estatal (sistema de clases), y d) económico societal (sistema de mercado). Una lectura del primero al último pauta una relación de constitución, y en una mirada a la inversa, es decir, desde lo económico societal a lo biocomunal, plantea un vínculo de regulación. En el caso de la investigación que aquí se presenta se puede observar la forma en que el sistema de mercado establecido en torno al agronegocio establece ciertos posicionamientos en la estructura de clases, y genera un sistema cultural que posibilita la convivencia cotidiana con un modelo que, a pesar de los cuestionamientos o reparos, es reconocido como fundamental en la reproducción material de la vida. Esto pauta las producciones subjetivas y el rol de los actores en las posibles transformaciones de las situaciones dañinas vinculadas con el uso de agroquímicos. Tal como fue señalado en la introducción de este artículo, en las comunidades se expresan una pluralidad de visiones, algunas de ellas tendientes a conservar lo establecido y otras dirigidas a su transformación (Fernández, 2007). El diseño y dispositivo metodológico instrumentado en este estudio permitió obtener una primera aproximación a estas orientaciones, donde la ubicación del actor en el proceso de producción y su rol en la reproducción social de la vida condiciona la dirección de las creaciones imaginarias. Esto es similar a lo observado en otros estudios realizados con comunidades urbano- agrícolas en Costa Rica (Barraza, et al., 2011) y en el sur de México (Ríos-González, et al., 2013).

En tercer lugar, otro nivel de análisis se vincula con los alcances de las miradas y perspectivas sobre los agroquímicos y la exposición colectiva a ellos. Es decir, qué se focaliza y qué aspectos se priorizan del problema. Los resultados permiten observar cierta tensión entre la preocupación única por la exposición a los agroquímicos y sus otros elementos concomitantes, y la interrogación sobre los aspectos estructurales del modelo productivo, donde estas sustancias constituyen uno de los medios de trabajo. Cuando los agroquímicos forman parte de una lógica productiva que construye territorialidades particulares y la población convive cotidianamente con ellos, el contacto ya no se presenta como una posibilidad que puede o no ocurrir como se plantearía desde el paradigma del riesgo (Almeida Filho, et al., 2009). Por el contrario, la convivencia con los agroquímicos se presenta de forma permanente, deja de ser una exposición eventual y se constituye como algo característico del modo de vida en estas agrociudades (Hernández, et al., 2012). Breilh (2003) distingue la exposición, como proceso eventual, crónico o diario, de la imposición, en tanto proceso continuo e inherente al modo de vida de un grupo o población. De este modo, la distinción principal entre los procesos de exposición eventual o crónica y los procesos de imposición es que los primeros tienen cierto grado de probabilidad de ocurrir o no, mientras que la imposición hace a un modo de vida, es decir, opera siempre, sin lugar para la aparición o no de su ocurrencia. De los distintos espacios de taller y discusión con actores sociales de Young surge la preocupación por la exposición a los productos, insumos y maquinaria utilizada en la producción; sin embargo, no se expresaron consideraciones sobre lo que subyace y hace posible la probabilidad de un daño a la salud, esto es, el modo de vida fomentado por el agronegocio. Al respecto, Breilh (2003) afirma que si se focaliza en los elementos contingentes, además de que se fragmenta el problema de los agroquímicos, esto solo puede devenir en soluciones temporales y parciales. La fragmentación en el abordaje del tema fue algo señalado por los actores sociales durante el estudio.

A modo de conclusión y como consideraciones finales es de interés detenerse en lo que estos resultados y su análisis aportan para generar transformaciones en las situaciones de vulnerabilidad derivadas de la exposición a los agroquímicos. Por un lado, la investigación desarrollada permite observar que la producción agrícola no solo involucra elementos agronómicos y económicos, sino que también genera un correlato simbólico, el cual produce significaciones particulares sobre el entorno y sus problemas. Denegar estos aspectos puede tornar inoperante cualquier alternativa que pretenda transformar prácticas agrícolas o generar medidas preventivas entre la población. Por otro lado, conocer esas significaciones sociales 
y su orientación en lo histórico social posibilita aproximarse a las condiciones necesarias, contradicciones y viabilidad de las propuestas que se diseñen. En el caso de la investigación general donde se enmarca este trabajo se lograron observar las condiciones necesarias para un monitoreo participativo en ambiente y salud, pero su viabilidad no pudo sostenerse más allá de la presencia del equipo universitario en el territorio (Abbate, et al., 2015). Por último, la instrumentación de dispositivos colectivos de investigación social en temas productivos genera condiciones de posibilidad y de visibilidad sobre aspectos que no habían sido observados desde una mirada ingenua o parcial de la realidad. La complejidad del fenómeno, en tanto proceso estratificado (Samaja, 2009), requiere de abordajes novedosos y de instrumentos que permitan entender sus múltiples determinaciones. Abordajes que motoricen prácticas transformadoras a distintos niveles o estratos de la realidad social.

\section{Reconocimientos}

Al Programa de Investigación e Innovación Orientado a la Inclusión Social de la Comisión Sectorial de Investigación Científica de la Universidad de la República, Uruguay. La investigación «Salud laboral y ambiental en torno a la utilización de agroquímicos en la ciudad de Young. Estrategias de monitoreo participativo» fue evaluada y financiada por este programa en su edición 2013- 2015. En este trabajo se presentó parte de lo relevado en la primera etapa de esta investigación.

Al Lic. en Geografía Andrés Fonsalía (1974- 2014) por su orientación, compromiso y dedicación con esta investigación.

\section{Referencias}

Abbate, S., Colazzo, M., Fonsalía, A., Heinzen, H., Heinzen, J., Niell, S., Pareja, L., Rodríguez, N. y Villar, A., 2015. Agroquímicos, salud laboral y ambiental: Diálogo de saberes $y$ búsqueda de alternativas en una comunidad urbana del litoral del país. Montevideo: CSIC.

ABRASCO, 2015. Dossiê ABRASCO: um alerta sobre os impactos dos agrotóxicos na saúde. Rio de Janeiro: EPSJV. ISBN: 978-85-9876-880-9

Alavanja, M. y Bonner, M., 2012. Occupational pesticide exposures and cancer risk: a review. En: Journal of Toxicology and Environmental Health, Part B: Critical Reviews, 15(4), pp.238-263.

Almeida Filho, N., Castiel, L.D. y Ayres, J.R., 2009. Riesgo: concepto básico de la epidemiología. En: Salud Colectiva, 5(3), pp.323-344.

Almeyra, G., Concheiro Bórquez, L., Mendes Pereira, J.M. y Porto-Gonçalvez, C.W., 2014. Capitalismo: tierra y poder en América Latina (1982-2012). México: CLACSO. ISBN: 978-607-28-0144-8.

Álvarez, E, 2014. Siglo deleuziano, siglo de los mapas: cualidades, sentidos y procesos puestos en juego en las cartografias de la subjetividad. En: Raega, 30, pp.11-40.

Arellano, E., Camarena, L., Von Glascoe, C. y Daesslé, W., 2009. Percepción del riesgo en salud por exposición a mezclas de contaminantes. El caso de los valles agrícolas de Mexicali y San Quintín. En: Rev. Fac. Nac. Salud Pública, 27(3), pp.291-301.
Ayres, J., Calazans, G., Filho, H. y Júnior, I., 2009. Risco, vulnerabilidade e práticas de prevenção e promoção da saúde. En: Campos, G., Minayo, M., Akerman, M., Júnior, M. y Carvalho, Y., org., 2009.Tratado de saúde coletiva. Sao Pablo: FIOCRUZ. pp.375- 418. ISBN: 9788527107044

Barraza, D., Jansen, K., Van Wendel de Joode, B. y Wesseling, C., 2011. Pesticide use in banana and plantain production and risk perception among local actors in Talamanca, Costa Rica. En: Environmental Research, 111, pp.708-717.

Blair, A., Ritz, B., Wesseling, C. y Beane, L., 2014. Pesticides and human health. En: Occupational and Environmental Medicine, 72(2), pp.1-2.

Blanco, J., 2010. Notas sobre la relación transporte-territorio: implicancias para la planificación y una propuesta de agenda. En: Transporte y Territorio, 3, pp.172-190.

Breilh, J., 2003. Epidemiología crítica. Buenos Aires: Lugar Editorial. ISBN: 950-892-147-1.

Castoriadis, C., 2013. La institución imaginaria de la sociedad. Barcelona: Tusquets. ISBN: 9788483834909.

Castro, M.J. y Confalonieri, U., 2005. Uso de agrotóxicos no Município de Cachoeiras de Macacu (RJ). En: Ciênc. Saúde Coletiva, 10(2), pp.473:482.

De Castro, C., 1997. La geografía en la vida cotidiana. De los mapas cognitivos al prejuicio regional. Barcelona: Ediciones del Serbal. ISBN: 978-84-7628-199-4

Fernández, A., 2007. Las lógicas colectivas. Buenos Aires: Biblos. ISBN: 978-950-786-575-6.

Guattari, F., 1996. Caosmosis. Buenos Aires: Manantial. ISBN: 978-987-500-006-3.

Gutiérrez-Strauss, A., González Baltazar, R., Salazar Estrada, J., Soltero Avelar, R., Aguilera Velasco, M. y León Cortés, S., 2013. Veneno para plagas: una aproximación desde la antropología cognitiva sobre exposición laboral, efectos en salud y calidad de vida de los aplicadores de plaguicidas del sector informal rural. En: Salud Uninorte, 29(3), pp.501-513.

Hernández, R., Fernández, C., Baptista, P., 2006. Metodología de la investigación. México: McGraw Hill Interamericana. ISBN: 970-10-5753-8.

Hernández, V., Fossa, M. y Muzi, M., 2012. Transformaciones productivas y perfiles sociales en la región pampeana a partir de un estudio comparativo. En: Estudios Rurales, 1(3), pp.220-255.

INE, 2011. Censos 2011 [En línea]. Montevideo: INE. [Consulta 15 de febero de 2017]. Disponible en: http:// www.ine.gub.uy/censos-2011

Mançano, B., 2013. Territorios: teoría y disputas por el desarrollo rural. En: Novedades en población, 17, pp.116-133.

Martínez, M., Morador, M., Peyrou, S., Mosquera, S., González, C., Díaz, L. y Cervieri, M., 2006. Tejiendo redes de salud comunitaria: agrotóxicos en Bella Unión. Montevideo: Redes Amigos de la Tierra. ISBN: 9974-7969-5-4

Menéndez, E., 2010. La parte negada de la cultura. Rosario: Pro Historia. ISBN: 978-987-1304-59-2.

Minayo, M.C.S., 2009. La artesanía de la investigación cualitativa. Buenos Aires: Lugar Editorial. ISBN: 978-950892-331-8.

Montero, M., 2006. Hacer para transformar. Buenos Aires: Paidos. ISBN: 950-12-4535-7.

Morina, J. y Cacace, G., 2013. Capitalismo agrario y expansión sojera en la Argentina. En: Meridiano - Revista de Geografía, 2, pp.105-126. 
Neme, C., Ríos, M., Zaldúa, N. y Cupeiro, S., 2010. Aproximación a la normativa vigente sobre plaguicidas $y$ sus impactos ambientales. Montevideo: Vida Silvestre. ISBN: 978-9974-7589-3-3.

Oyhancabal, G. y Narbondo, I., 2009. Radiografia del agronegocio sojero. Montevideo: REDES-AT. Uruguay. ISBN: 978-9974-7885-8-9

Palau, M., 2015. Con la soja al cuello. Asunción: BASE. ISBN: 978-99967-788-4-1

Peres, F. y Moreira, J.C., 2003. É veneno ou é remédio?: agrotóxicos, saúde e ambiente. Rio de Janeiro: FIOCRUZ. ISBN 85-7541-031-8

Rance, S. y Tellería, J., 2003. Saliendo del anonimato: el mapeo de actores/as sociales como instrumento para el diseño de políticas. En: Reunión regional. Sexualidades, salud y derechos humanos en América Latina. Lima: Universidad Peruana Cayetano Heredia.

Reygadas, R., 1998. Abriendo veredas. Iniciativas públicas y sociales de las redes de organizaciones civiles. México DF: UAM.

Ríos, M., Zaldúa, N. y Cupeiro, S., 2010. Evaluación participativa de plaguicidas en el sitio RAMSAR, Parque Nacional Esteros de Ferrapos e Islas del Río Uruguay. Montevideo: Vida Silvestre. ISBN: 978-9974-7589-5-7.

Ríos-González, A., Jansen, K. y Sánchez-Pérez, H., 2013. Pesticide risk perceptions and the differences between farmers and extensionists: towards a knowledge-in-context model. En: Environmental Research, 124, pp.43-53.
Ritter, L., Goushleff, N., Arbuckle, T., Cole, D. y Raizenne, M., 2006. Addressing the linkage between exposure to pesticides and human health effects- research trends and priorities for research. En: Journal of Toxicology and Environmental Health, 9, pp.441-456.

Rodríguez, N., 2017. Producción subjetiva sobre la exposición a agroquímicos. Revisión de la bibliografía científica. En: Ciênc. Saúde Coletiva, en prensa.

Rulli, J., 2009. Pueblos fumigados: los efectos de los plaguicidas en las regiones sojeras. Buenos Aires: Del Nuevo Extremo. ISBN: 9876091697.

Samaja, J., 2009. Epistemología de la salud. Buenos Aires: Lugar Editorial. ISBN: 978-950-892-189-5.

Stake, R.E., 1999. Investigación con estudio de caso. Madrid: Morata. ISBN: 84-7112-422-X.

Uruguay. Decreto 379/008, de 4 de agosto de 2008. Diario Oficial, 14 de agosto de 2008, No. 27.547, p.415A.

Vargas Trejos, Y., 2015. Exposición a agroquímicos y creencias asociadas a su uso en la cuenca hidrográfica del Río Morote, Guanacaste, Costa Rica. Un estudio de casos. En: Ciencia\&Trabajo, 17(52), pp.54-68.

Vasilachis, I., 2007. Estrategias de investigación cualitativa. Buenos Aires: Gedisa. ISBN: 978-84-9784-173-3.

Ye, M., Beach, J., Martin, J. y Senthilselvan, A., 2013. Occupational pesticide exposures and respiratory health. En: Environmental Research and Public Health, 10, pp.6442-6471. 